\title{
CMRegNet-An interspecies reference database for corynebacterial and mycobacterial regulatory networks
}

\author{
Vinicius A. C. Abreu ${ }^{1 *}$, Sintia Almeida ${ }^{1}$, Sandeep Tiwari ${ }^{1}$, Syed Shah Hassan ${ }^{1}$, Diego Mariano ${ }^{1}$, Artur Silva ${ }^{2}$, \\ Jan Baumbach ${ }^{3}$, Vasco Azevedo ${ }^{1}$ and Richard Röttger ${ }^{3,4^{*}}$
}

\begin{abstract}
Background: Organisms utilize a multitude of mechanisms for responding to changing environmental conditions, maintaining their functional homeostasis and to overcome stress situations. One of the most important mechanisms is transcriptional gene regulation. In-depth study of the transcriptional gene regulatory network can lead to various practical applications, creating a greater understanding of how organisms control their cellular behavior.

Description: In this work, we present a new database, CMRegNet for the gene regulatory networks of Corynebacterium glutamicum ATCC 13032 and Mycobacterium tuberculosis H37Rv. We furthermore transferred the known networks of these model organisms to 18 other non-model but phylogenetically close species (target organisms) of the CMNR group. In comparison to other network transfers, for the first time we utilized two model organisms resulting into a more diverse and complete network of the target organisms.

Conclusion: CMRegNet provides easy access to a total of 3,103 known regulations in C. glutamicum ATCC 13032 and M. tuberculosis H37Rv and to 38,940 evolutionary conserved interactions for 18 non-model species of the CMNR group. This makes CMRegNet to date the most comprehensive database of regulatory interactions of CMNR bacteria. The content of CMRegNet is publicly available online via a web interface found at http://lgcm.icb.ufmg.br/cmregnet.
\end{abstract}

\section{Background}

The ever growing number of completed genome sequencing projects has allowed for the extensive use of computational approaches for comparative genomics identifying potential transcriptional regulatory networks and key elements, such as transcription factor binding sites (TFBSs) [1]. These studies primarily focus on analyzing and describing regulatory elements that have been previously identified in model organisms and how this information may be applicable to organisms that have yet to be characterized. The comparative analysis of regulators combined with other genomic-context analysis techniques significantly improves the quality and accuracy of the functional

\footnotetext{
* Correspondence: vabreu@isoladas.grad.ufmg.br; roettger@mpi-inf.mpg.de ${ }^{1}$ Graduate Program in Bioinformatics, Institute of Biological Sciences, Federal University of Minas Gerais (Universidade Federal de Minas Gerais), Belo Horizonte, Minas Gerais, Brazil

${ }^{3}$ Department of Mathematics and Computer Science, University of Southern Denmark, Odense, Denmark

Full list of author information is available at the end of the article
}

gene annotations and the predictions of genes that may be involved in a variety of regulatory networks [2].

It is currently not possible, however, to decipher a complete regulatory network, even for a model organism. Potential reasons for this include an inability to simulate the different environmental conditions in which the organism lives in the laboratory and the inherent background noise of the existing wet-lab techniques. Even for the model organism E. coli, only a third of its transcriptional regulatory network (TRN) has been identified, even though a large number of studies with accurate data have been published on this organism [3]. The situation even worsens, when we focus on organisms like Mycobacterium leprae, which is the bacterium with the longest known duplication time and which does not grow in culture medium [4]. The aim of this study was to qualitatively and quantitatively contribute to the reconstruction of the transcriptional regulatory network between phylogenetically related species, specifically for species belonging to the CMNR group. The CMNR group belongs to the family of 
actinomycetes and consists of organisms belonging to the genera Corynebacterium, Mycobacterium, Nocardia, and Rhodococcus. Their phylogenetic correlation has been confirmed by $16 \mathrm{~S}$ rDNA and rpoB DNA sequences analyses. The members of this group are Gram-positive bacteria that exhibit many peculiar features: (i) high $\mathrm{G}+\mathrm{C}$ content, and (ii) a specific organization of the cell wall composed of mycolic acid, peptidoglycan and arabinolactano [5]. This group consists of several bacterial species that are of medical, veterinary, and biotechnological interest. Furthermore, some species of the CMNR group are important for industrial and biotechnological applications, such as Corynebacterium glutamicum and Corynebacterium efficiens [6]. In contrast, pathogenic species such as Mycobacterium tuberculosis and Corynebacterium diphtheriae (causing tuberculosis and diphtheria in humans, respectively) and C. pseudotuberculosis, which infects various animal species, especially small-size ruminants, are also prominent members of this group [7]. Because of their importance, several genomes of the CMNR group have been sequenced.

In the framework of this study, we aimed to computationally transfer the knowledge of the known TRN of the two-model organisms Corynebacterium glutamicum ATCC 1303 and Mycobacterium tuberculosis H37Rv to 18 other organisms of the CMNR group. The results were stored in an ontology-based database and are publicly available through the online platform CMRegNet. The platform also allows for several types of queries to access the database content and supports the reconstruction, analysis and visualization of the regulatory networks at different hierarchical levels.

CMRegNet is an interactive analysis platform for studying the transcriptional regulatory networks of the CMNR group of bacteria. The platform is publicly available at http://gcm.icb.ufmg.br/cmregnet.

\section{Construction and content}

\section{Overview of the CMRegNet system}

The CMRegNet system is a database for transcriptional gene regulatory interactions of 20 (2 model organisms, 18 target organisms) different strains of the genera Corynebacterium and Mycobacterium. The system incorporates several bioinformatics data analysis procedures and information from different sources, in order to provide the user with all relevant information on regulatory interactions including the binding sites, protein sequences, gene annotations, and the genomic context of the regulation. The database itself runs on a MySQL 5.5 community server. The web service of CMRegNet is written in PHP (version 5.2.1) and delivered by an Apache web server (version 2.2.22).

As aforementioned in the introduction, the amount of known regulations is very scarce and only limited to a handful of model organisms. Thus, one key aspect of CMRegNet is the automated transfer of evolutionarily conserved regulations of these model organisms to the so called target organisms. For CMRegNet we exploit the same transfer pipeline which was already successfully used in CoryneRegNet [8-12] and MycoRegNet [13] in order to predict evolutionarily conserved regulations. CMRegNet may be regarded as the successor of the discontinued MycoRegNet [13] but was significantly extended: (1) we utilized ChiP-Seq data of Mycobacterium tuberculosis $\mathrm{H} 37 \mathrm{Rv}$ in order to receive a comprehensive list of binding sites allowing $M$. tuberculosis to act as a model organism for the network transfer and (2) in contrast to comparable systems, CMRegNet bases the transfer of evolutionarily conserved regulations on two model organisms (Corynebacterium and Mycobacterium). This increases the predicted regulations in both, quantitative and qualitative aspects.

In order to transfer a regulation from a model organism to a target organism, we defined a simplified model of a gene regulation: A regulation requires three main drivers, namely the transcription factor, the target gene and the corresponding binding site in the upstream region of the target gene. We consider a regulation as evolutionarily conserved, if in the target organism (1) the transcription factor is conserved, (2) the target gene is conserved and (3) the target gene possesses the binding site for the transcription factor in its upstream region.

In order to detect conserved genes (i.e., homologous genes), we decided to perform a homology detection based on the clustering of the protein sequences, i.e., the reported clusters form the groups of homologous proteins. Generally, for the homology detection with a clustering tool, a similarity measure between the proteins and a meaningful parameter setting for the employed clustering tool are required. For CMRegNet we use transitivity clustering (TransClust) and followed the approach described in [14] which suggest the usage of a BLAST all-vs.-all run on the protein sequences with an E-value cut-off of 10 as similarity function. The threshold (the parameter of TransClust) was selected following the suggestions in [15]. In this study, the authors developed a measure for judging the quality of a clustering for homology detection by basically evaluating two aspects of the cluster-size distribution: (1) the number of genes in the core-genome (genes shared by all organisms) and (2) the number of unrealistically large clusters (which most likely contain false positives). The idea is now to find that threshold which maximizes (1) while minimizing (2). In the original study, the authors suggest to pick a threshold between 34 and 61 for mycobacteria and 27 and 53 for corynebacteria. For CMRegNet, we decided to use a rather non-stringent threshold of 30 which is in the middle of the two lower bounds of both suggested threshold ranges. We decided 
to do so because we (1) have proteins from both genera, Corynebacterium and Mycobacterium and (2) the homology detection is only one of three criteria (as described above) for the prediction of an evolutionarily conserved regulation. Thus we are convinced that this selection of the threshold does not increase the false-positive rate while providing a large basis of potential homologous proteins for the regulation transfer.

We used PoSSuMsearch [16] with a p-value cut-off of 10 in order to identify possible binding sites in the upstream region $(-540 \mathrm{pb} \ldots+40 \mathrm{pb}$ relative to start codon $)$ of the potential target gene. With that information, we can identify evolutionarily conserved regulations in the target organisms.

The transferred regulations undergo an additional refinement process utilizing operon predictions obtained from MicrobesOnline [17]. A regulation is only considered conserved, if the target gene is also the first gene in an operon. If this condition holds, all genes in the operon of the target organism are consequentially predicted to be regulated by the transcription factor in question.

In the case that the same regulation is predicted by the network transfer of both model organisms, we store and display two in silicio evidences for this regulation and refer to the two experimental validated regulations in the model organisms.

To sum up, for the target organisms, we require gene annotations and the operon predictions. For the model organisms we additionally need information of the regulatory interactions including the binding sites of the involved transcription factors. In the following, we describe all utilized data sources for CMRegNet.

\section{Target organisms}

For the 18 target organisms included into CMRegNet, the publicly available sequences and annotation data from the National Center for Biotechnology Information (NCBI) were retrieved [18]. The operon prediction data was provided by an integrated portal for comparative and functional genomics, MicrobesOnline [17].

\section{Model organisms}

For both model organisms, we obtained the operon predictions as well as the gene annotations from the same sources as for the target organisms. For the model organisms, additional information on the regulatory interactions had to be derived. The reconstruction of both regulatory networks is mainly composed of experimental data derived from the literature. In the following section, we describe the additional data sources used.

\section{Corynebacterium glutamicum ATCC 13032}

With CoryneRegNet [12], there already exists a reference database and analysis platform for corynebacterial gene regulatory networks. The biological content of CoryneRegNet comprehensively covers transcriptional regulations in the model organism C. glutamicum ATCC 13032 and provides all necessary information for CMRegNet, inclue TFBS and regulation. We extracted a total of 1,441 known regulatory interactions, 520 TFBS, 97 regulators, and their respective target genes. The data of CoryneRegNet is derived from various wet-lab experiments such as ChiPChiP, ChiP-Seq, and microarrays, but mostly derived from microarray experiments [8].

\section{Mycobacterium tuberculosis $\mathrm{H} 37 \mathrm{Rv}$}

For M. tuberculosis H37Rv, despite being a wellestablished model organism, no such database providing necessary support for transcriptional gene regulatory networks exists. However, for M. tuberculosis H37Rv, the Tuberculosis Database (TBDB) serves as a database collecting all tuberculosis related research resources, e.g., expression data, metabolomic networks, relevant publications, and many more. Especially, TBDB hosts several omics data from multiple strains of $M$. tuberculosis, as well as data related to the genera Mycobacterium $[16,19]$. In contrast to C. glutamicum, we do not have the TFBS information of each mapped regulator for the genome of M. tuberculosis H37Rv. However, TBDB provides for every regulator the upstream region of the target genes which most likely contain the TFBS. In order to extract the actual binding sites required for CMRegNet, we performed the following strategy.

\section{Retrieving the binding sites for $M$. tuberculosis}

Through its "Search Regulatory Binding Sites" option, the TBDB provides a table of possible regulatory genes for a given gene of interest (Fig. 1a). The information is based on ChIP-Seq experiments. We processed the following core information: (1) gene regulator, (2) the distance of start codon of the target gene, and (3) the start and stop coordinates of a region of possible TFBS.

However, to predict TFBS in these regions, we found some inconsistencies, such as overlap, distance, size and peaks, within the operons (Fig. 1a and 1c). Although, there are some reported cases where the TFBS is found in regions with a high overlap and more significant distances in M. tuberculosis [20], we followed a more stringent criterion to reduce the number of false positives. We limited the peak regions to an area between +40 bps to $-540 \mathrm{pbs}$ in relation to the target gene. A Perl script was used to filter the data obtained by TBDB. For each regulator a FASTA file consisting of all sequences possibly containing the TFBS was created (Fig. 1d). These FASTA files formed the input for a subsequent TFBS prediction using MEME-ChIP [21].

MEME-Chip is a tool used for predicting largescale motif sequences. We performed a MEME run 


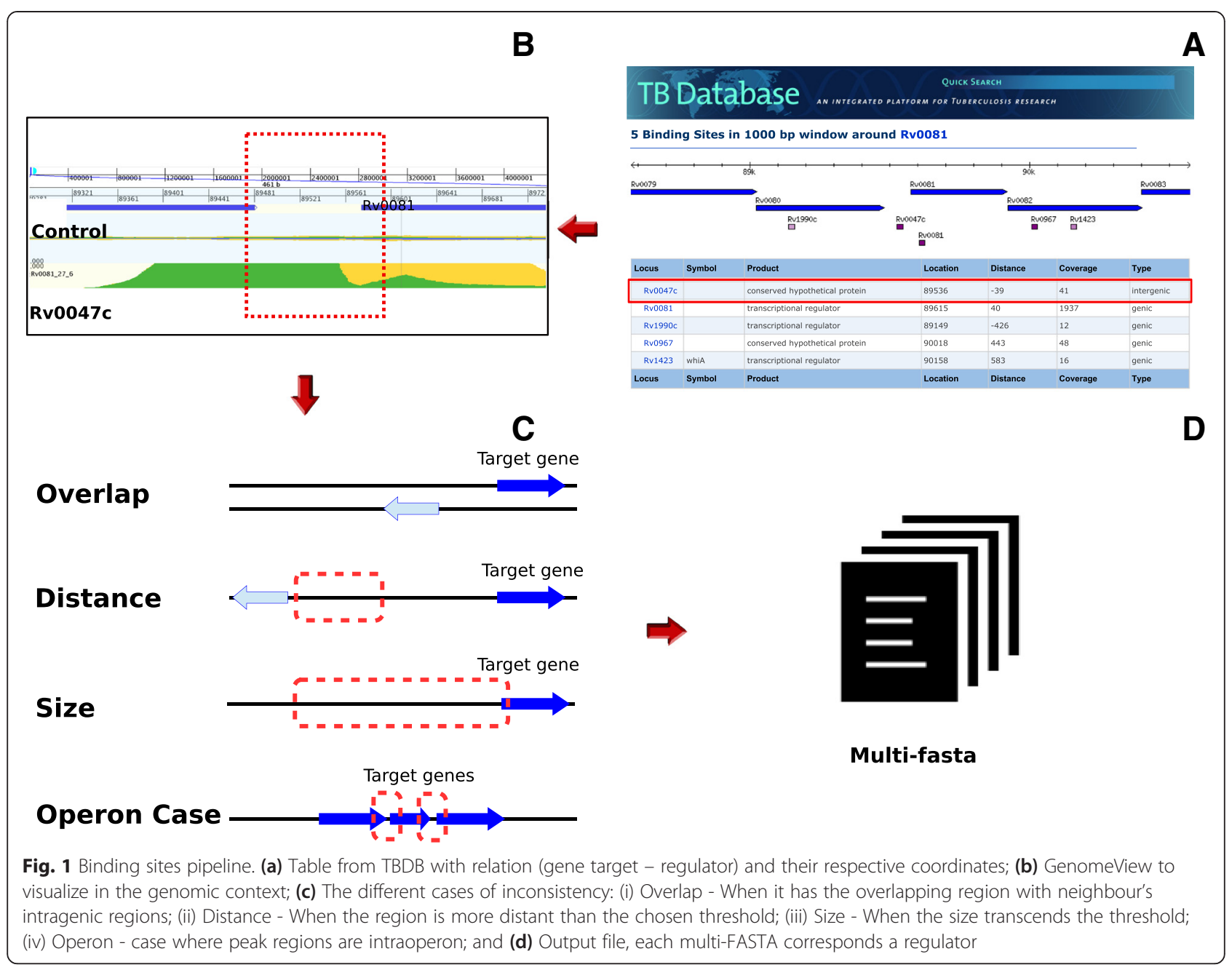

on each FASTA file using the default parameters. An extensive literature search was performed to look for experimental data on TFBS. Whenever experimental evidence for a TFBS was available, we utilized this additional information by becoming more stringent in the setting of the "Maximum width motif" parameter according to the motif reported in the literature. An overview of the pipeline analyses is depicted in Fig. 2.

At this point, we have acquired all required data of the model organisms, namely the set of regulators and target genes with their corresponding TFBS. With this information we are able to run the previously described network transfer pipeline and transfer all evolutionarily conserved regulation from both model organisms to all 18 target organisms.

\section{Utility and Discussion}

The utilization of experimental data of two phylogenetically close model organisms (C. glutamicum and M. tuberculosis) combined with the comparative genomics approach for transferring the gene regulatory network makes the CMRegNet a reference database for studying regulatory networks of organism in the CMNR group. Table 1 summarizes the database content of CMRegNet. The CMRegNet is divided into two evidence levels: (1) experimental and (2) predicted. The experimental version only contains experimentally validated regulations whereas the predicted version additionally contains the transferred evolutionarily conserved regulations as well. In Table 1, we depict all regulations stored in the predicted version of CMRegNet. Whenever a regulation was predicted as conserved based on both model organisms, it was counted as two regulations in the table.

So far, databases similar to CMRegNet were limited to only one model organism, e.g., in [12, 22]. Considering the scarce knowledge we have on regulatory interaction even for heavily studied model organisms [3], the restriction to one model organism poses one of the most prominent limitations of the automated network transfer [23]. Apparently, a regulation can only be transferred to a target organism, when it was experimentally validated 


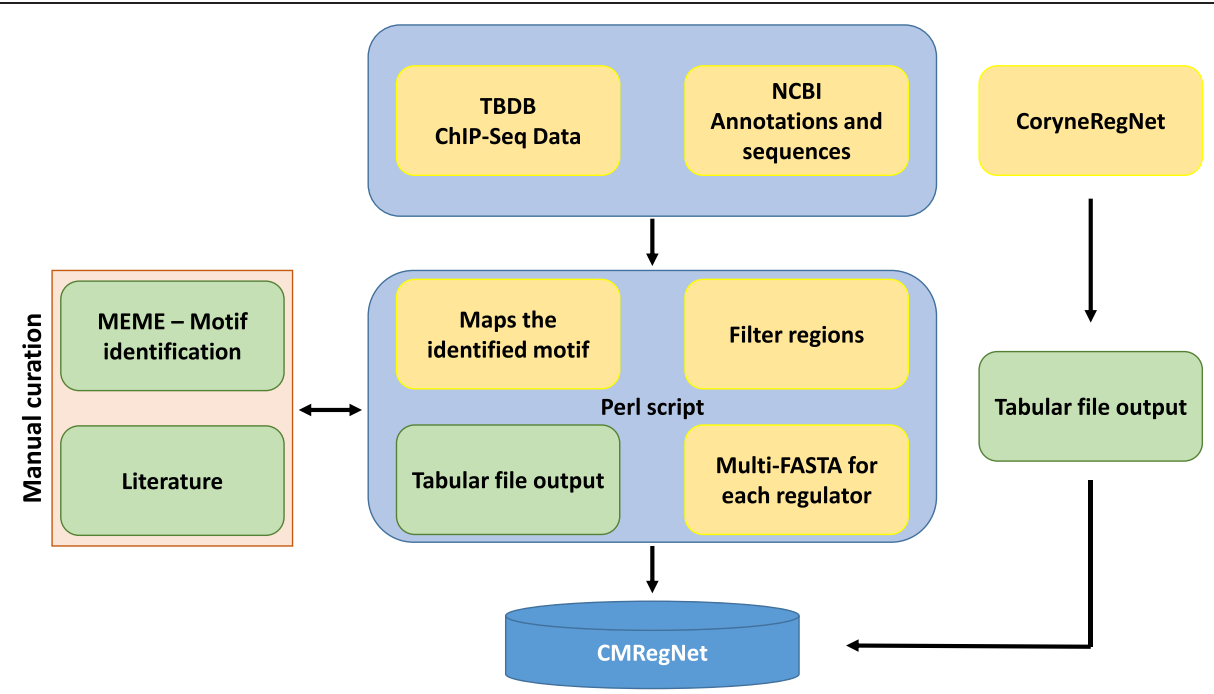

Fig. 2 Schematic overview illustrating the retrieval of binding sites information of both model organisms M. tuberculosis H37Rv and C. glutamicum ATCC 13032 using TBDB and CoryneRegNet as main sources

in model organisms in the first place. Here, for the first time we utilized two model organisms which are phylogenetically close but with different life-styles. This allows us to overcome the limitations imposed by the use of a single model. We illustrate the potential of CMRegNet for Mycobacterium leprae, an etiologic agent of leprosy disease. Note, that $M$. leprae has a atypical genome within the CMNR group: a large number of pseudogenes, accumulation of insertion sequence, lowered $\mathrm{G}+\mathrm{C}$ content, which all are hallmarks of reductive evolution, and may reflect passage through an evolutionary bottleneck [24]. Nevertheless, CMRegNet was able to transfer 641 conserved interactions in total, 107 of them from C. glutamicum, 326 from $M$. tuberculosis, and 208 were conserved in both models. This is a strong indicator for the power and utility of using two model organisms instead of only one.

All large-scale integrative databases, such as CMRegNet, are facing the same challenge of integrating data from various sources derived by different techniques. The literature data from C. glutamicum ATCC 13032 shows a great diversity of techniques applied to study gene regulations. Exemplarily, the characterization of the global gene regulator glxR varies from ChIP-Seq techniques [25] over various studies of the gene transcription [26-29] to analyses derived from microarray and PCR experiments.

In contrast to C. glutamicum ATCC 13032, the data from M. tuberculosis H37Rv utilized in CMRegNet originates mostly from the "Flag-tagged" ChIP-Seq data. This approach allows studying a large number of transcription factors, without the necessity of previous knowledge of the conditions that normally induce its expression, and the identification of regions enriched in SLFT [20].
This enabled the usage of $M$. tuberculosis as a model organism in the first place.

The difference in the data sources between both model organisms is consequentially also reflected in the number of detected regulations (compare Table 1): 94 regulators responsible for a total of 1314 regulations are found in C. glutamicum whereas 40 regulators in $M$. tuberculosis are responsible for 2466 regulations.

In order to reflect this diversity of evidence in the provided data, every regulation stored in CMRegNet is linked to the source of its evidence so that researchers are able to make an informed decision whether a certain regulation is reliable enough or not for their purpose.

Furthermore, the automated transfer of evolutionarily conserved regulations has a main limitation: Only regulations already known in model organisms can possibly transferred to the target organisms. Additionally, even for these highly studied model organisms, the currently known regulatory network is far from complete [3]. Until this work, the databases similar to CMRegNet were limited only to one model organism [8-12, 30-32] which was extended to a second model organism for CMRegNet; but nevertheless the amount of information of the target organisms is strictly limited by the available information on the model organisms.

\section{Navigation}

CMRegNet is accessible by a user-friendly online interface. As already mentioned, CMRegNet provides the user with two different choices of evidence level: (I) experimental, which concentrates on the dataset with experimental verification, and (II) predicted, which additionally provides 
Table 1 This table summarizes the database content of CMRegNet

\begin{tabular}{|c|c|c|c|c|c|c|c|c|}
\hline Organism & Genes & Reg & TG & TFBS & CG & MT & Both & NRs \\
\hline C. aurimucosum ATCC 700975 & 2531 & 31 & 448 & 420 & 294 & 264 & 108 & 666 \\
\hline C. diphtheriae NCTC 13129 & 2272 & 37 & 379 & 470 & 270 & 68 & 210 & 548 \\
\hline C. efficiens YS-314 & 2950 & 44 & 601 & 537 & 443 & 262 & 248 & 953 \\
\hline C. glutamicum ATCC 13032 & 3058 & 94 & 723 & 452 & 1314 & 0 & 0 & 1314 \\
\hline C. glutamicum R & 3052 & 51 & 724 & 773 & 638 & 282 & 312 & 1232 \\
\hline C. jeikeium K411 & 2104 & 25 & 418 & 440 & 212 & 156 & 223 & 591 \\
\hline C. kroppenstedtii DSM 44385 & 2018 & 24 & 399 & 321 & 199 & 114 & 279 & 591 \\
\hline C. pseudotuberculosis 1002 & 2057 & 31 & 408 & 339 & 285 & 91 & 239 & 615 \\
\hline C. pseudotuberculosis C231 & 2053 & 29 & 409 & 338 & 281 & 93 & 234 & 608 \\
\hline C. pseudotuberculosis FRC41 & 2110 & 30 & 437 & 355 & 303 & 93 & 250 & 646 \\
\hline C. urealyticum DSM 7109 & 2024 & 25 & 377 & 356 & 176 & 132 & 262 & 570 \\
\hline M. abscessus & 4920 & 45 & 1374 & 1855 & 198 & 2156 & 541 & 2895 \\
\hline M. avium 104 & 5120 & 40 & 1489 & 2358 & 101 & 2676 & 467 & 3244 \\
\hline M. bovis BCG str. Pasteur $1173 \mathrm{P} 2$ & 3948 & 53 & 2195 & 4117 & 115 & 5209 & 407 & 5731 \\
\hline M. leprae $\mathrm{Br} 4923$ & 1604 & 17 & 450 & 335 & 107 & 326 & 208 & 641 \\
\hline M. marinum M & 5423 & 44 & 1919 & 3805 & 121 & 4190 & 742 & 5053 \\
\hline M. smegmatis str. MC2 155 & 6717 & 50 & 1555 & 2299 & 266 & 2366 & 614 & 3246 \\
\hline M. tuberculosis F11 & 3941 & 52 & 1934 & 3988 & 106 & 4852 & 294 & 5252 \\
\hline M. tuberculosis H37Ra & 4034 & 54 & 2380 & 4391 & 142 & 5654 & 498 & 6294 \\
\hline M. tuberculosis H37Rv & 4003 & 40 & 1458 & 2466 & 0 & 2466 & 0 & 2466 \\
\hline
\end{tabular}

Legend: Genes = total number of genes; Reg = total number of regulatory genes; $T G=$ number of target genes; TFBS = total number of binding sites; $C G=$ number of regulations exclusively transferred from Corynebacterium glutamicum ATCC 13032; MT = number of regulations exclusively transferred from Mycobacterium tuberculosis H37Rv; Both = regulations predicted by both model organisms; NRs = total number of regulations

the transferred regulations. After selecting the evidence level, the user is presented an overview page consisting of a list of organisms, summary statistics, and search box for almost arbitrary queries.

In the following, we demonstrate the power of CMRegNet using the gene Rv0081 as an example. The Rv0081 gene of $M$. tuberculosis $\mathrm{H} 37 \mathrm{Rv}$ encodes a transcriptional regulator HTH-type, a member of the dormancy regulon. The gene was first extensively described by Black et al. [33]. Rv0081 is of biotechnological importance and serves as an immunogenic antigen, inducing interferon-gamma, indicating that this might be a good vaccine candidate. It is hoped that this regulon will give insight into the latent or dormant phase of infection [33].

After identifying the genome and the gene of interest, the user receives all relevant information of the gene and its embedding in the regulatory network (Fig. 3). The results are displayed in an expandable list. In these sections, users can retrieve the following information: (a) the context of the gene in regard to the genome, (b) gene information, (c) protein information, (d) regulating genes and in case of a transcription factor additionally (e) a list of its target genes and (f) information about the corresponding binding site.
Furthermore, the user can search in the upstream region of the gene of interest for potential binding sites and-in case of transcription factors-search in the upstream region of all other genes for its associated binding site. In Fig. 3, it can also be observed that the genes that are part of the $R v 0081$ operon are highlighted in "light green". The CMRegNet allows clicking on any of the gene, which immediately redirect to another page with details of another gene.

CMRegNet also provides visualization of the network using GraphVis, which is a Java applet. The user can either display the whole regulatory network of the organism, or only the relevant part for a certain gene of interest. GraphVis allows the user to zoom into the chart, apply different layout styles, remove, add and edit selected elements (e.g., researchers can add new regulations of their current study in the visualization) or obtain detailed information about the selected genes. Figure 4 depicts the regulatory network of Rv0081.

Furthermore, GraphVis allows for the projection of expression levels onto the currently loaded network. This data can either be chosen from the integrated stimulon database or manually added. In Fig. 5, we manually added the gene expression results presented in [22]. In this work, Fontán et al. observed that after infecting 


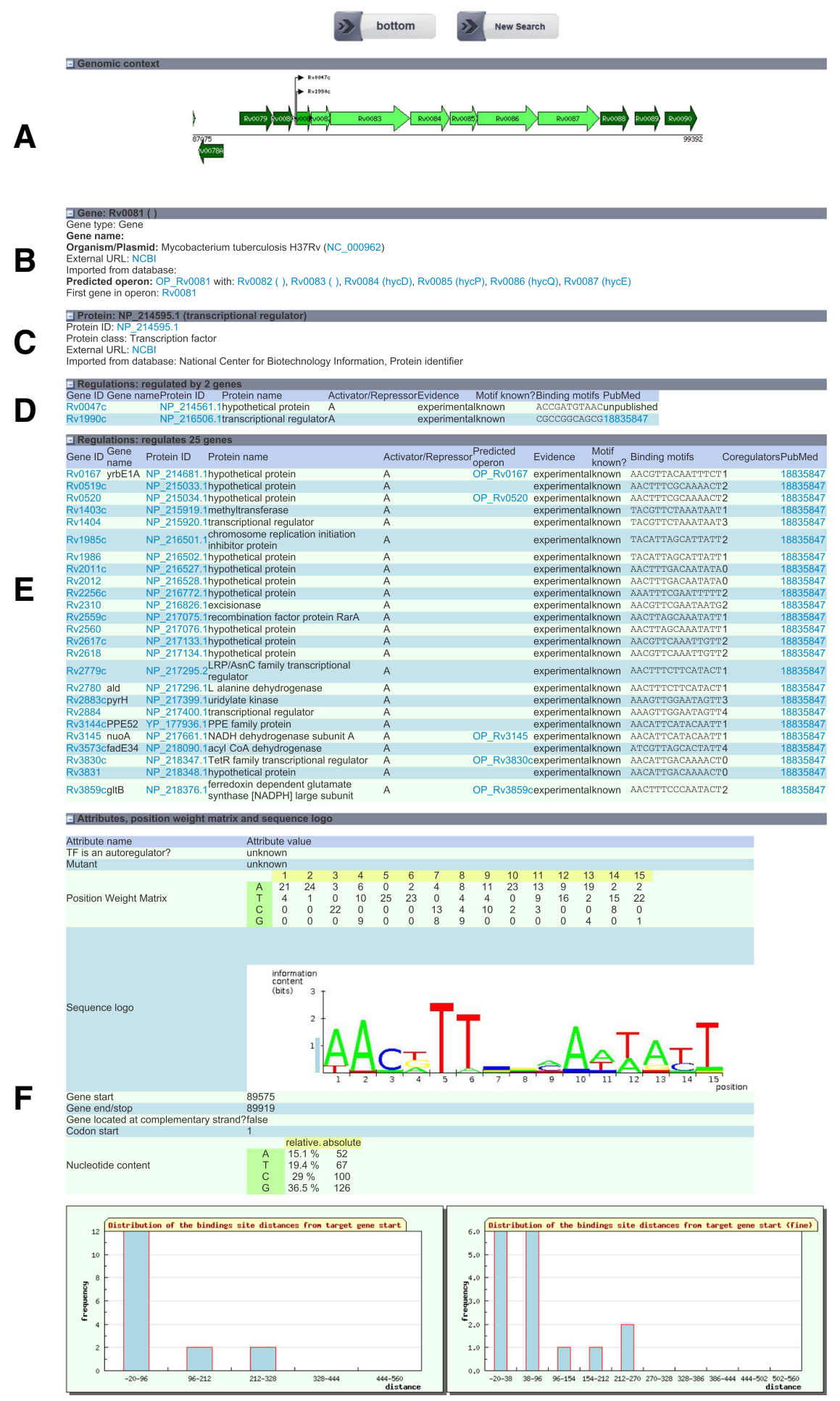

Fig 3 (See legend on next page.) 
(See figure on previous page.)

Fig 3 The expandable list summarizes the relevant data for the reconstruction of regulatory networks of the gene Rv0081 of $M$. tuberculosis. (a) The operon membership of the selected gene; (b) gene/protein information, providing links to the genome annotation deposited at the NCBI; (c/d/e) regulations, summarizing all available information on the gene regulatory network of the selected gene; (f) attributes, providing PSSMs and sequence logos of the predicted Rv0081 binding sites consensus. The height of each letter within an individual stack represents the nucleotide's frequency relative to the particular motif position; thus, the stack according to the respective position indicates the degree of a nucleotide's conservation

IFN- $\gamma$-treated BMM-differentiated THP-1 cells, 11 genes were also up regulated, namely: Rv0080, Rv0081, Rv2028c, pfkB, Rv2030c, acr, acg, Rv2626c, Rv3133c, fadE24, and $f a d E 23$. Such information can easily be integrated and visualized by GraphVis.

As a second example demonstrating the strength of CMRegNet, we present the findings of the gene $g l x R$. This gene is a DNA-binding transcriptional regulator of the FNR-CRP protein family and is well studied in genus Corynebacterium $[26,28]$. However, this gene is only little studied in the genus Mycobacterium, with no available information on regulations of this gene so far. For example, in $M$. abscessus the gene MAB_0416c was identified as a homologous protein to $g l x R$ and through the network transfer, CMRegNet now shows 102 conserved regulations.

The analysis of the Rv0081 and $g l x R$ gene demonstrated the in-depth analysis capabilities of CMRegNet for gene regulatory networks.

\section{Conclusion}

In this work, we have reconstructed the regulatory network of two important pathogen models of the genera Corynebacterium and Mycobacterium, including publicly available experimentally validated data. This data has been computationally transferred to 18 related organisms (see Table 1), making it so far the largest

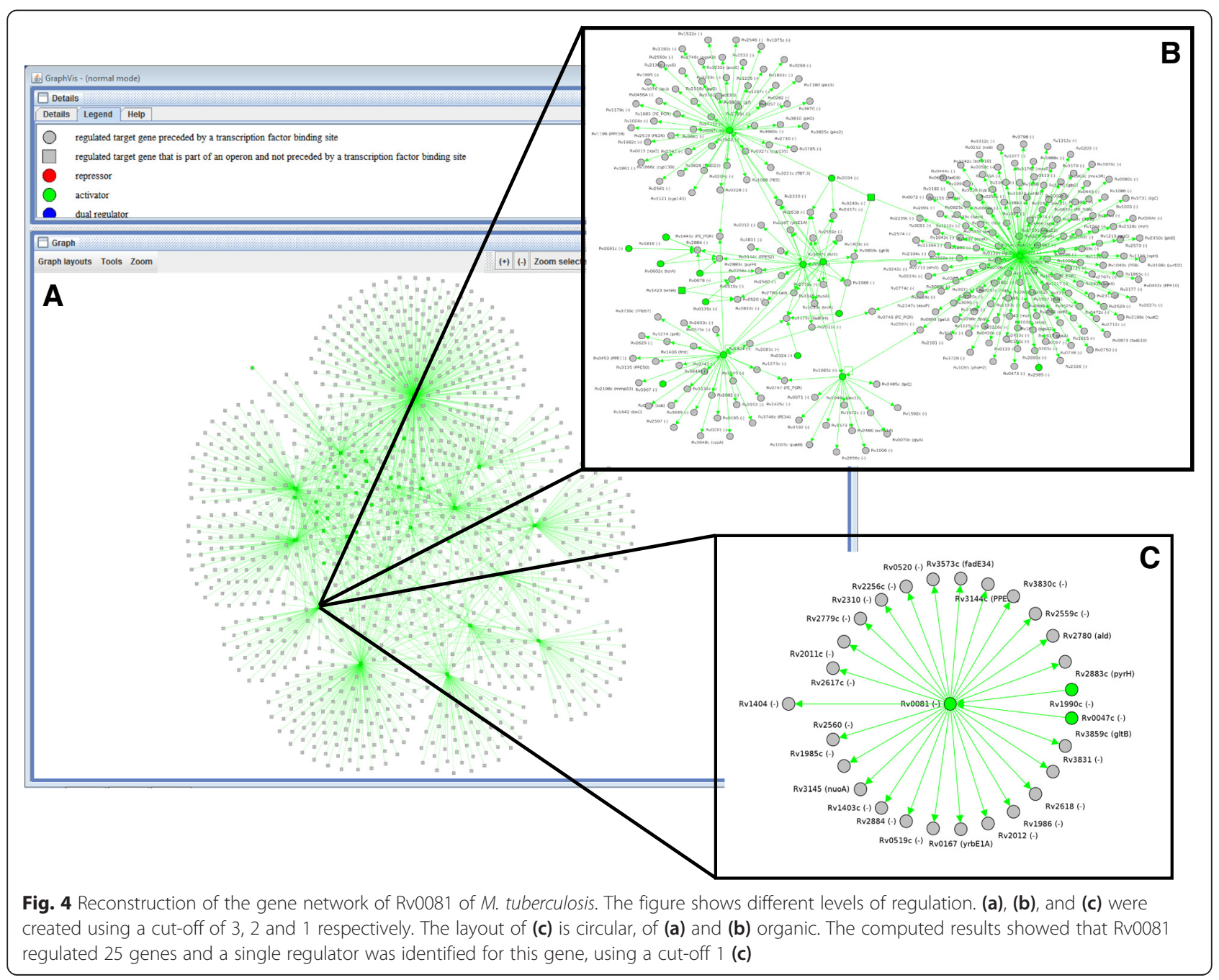




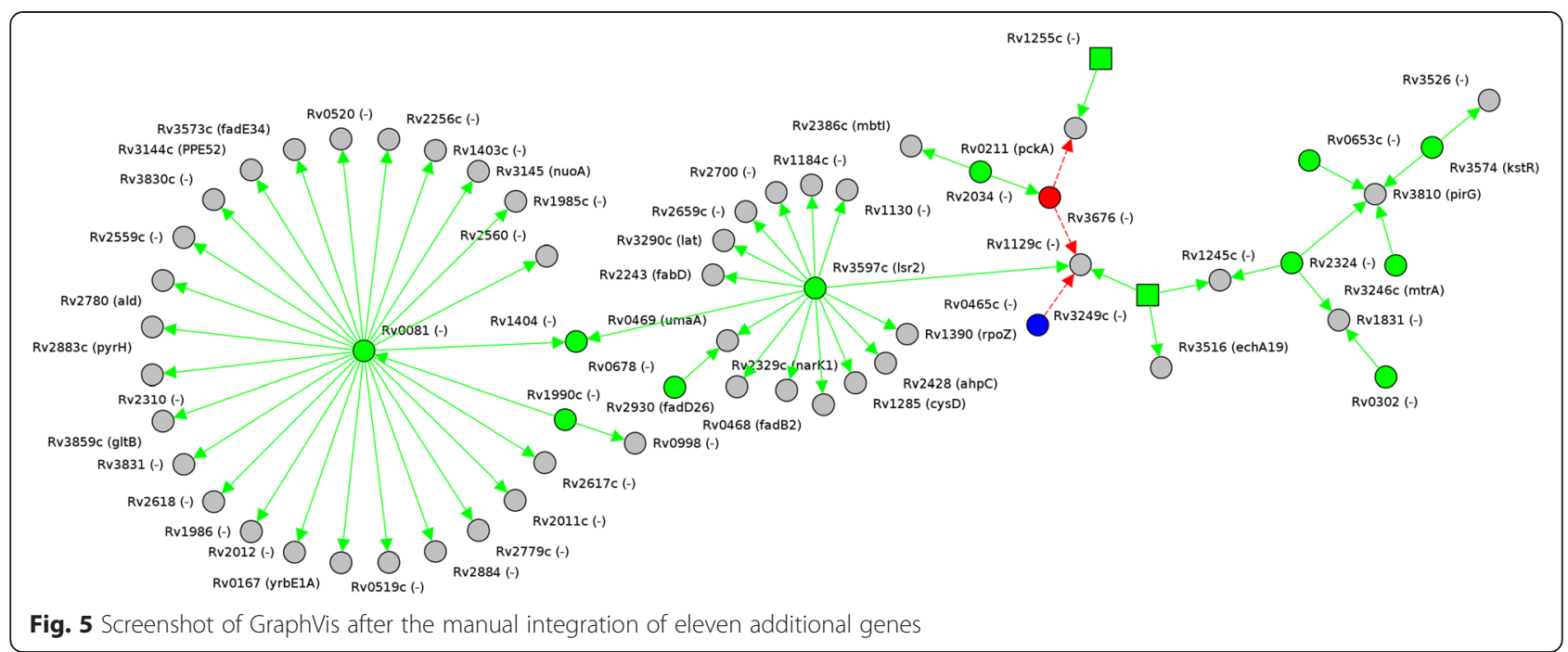

database of regulatory network targeted to the CMNR group. CMRegNet provides the data in an easily accessibly manner allowing for efficient analyzes of the regulatory networks and furthermore provides the user with the possibility to integrate own data into the analysis. These features support researchers in designing their future wet-lab experiments.

Furthermore, we constantly screen the relevant literature and regularly extend the database with newly published regulations.

\section{Availability and requirements}

The CMRegNet database is freely available for noncommercial use at http://lgcm.icb.ufmg.br/cmregnet. The GraphVis applet requires a browser with a Java plug-in installed.

\section{Competing interests}

The authors declare that they have no competing interests.

\section{Authors' contributions}

The in silico analyses were carried out by SA, AS, and VACA, the identification of the binding site predictions was performed by ST, SSH, and VACA. VACA, $\mathrm{RR}$, and JB designed and implemented the back-end, DCBM designed the front-end. JB and RR drafted the paper, VACA, SA, and RR wrote the final version of the manuscript. SSH, AS, VA contributed through consultation and extensive manuscript reviewing. VA, RR and JB designed and coordinated the project together. All authors read and approved the final manuscript.

\section{Authors' information}

Jan Baumbach, Vasco Azevedo and Richard Röttger are joint last authors.

\section{Acknowledgement}

RR is grateful for funding from the International Max Planck Research School in Computer Science as well as from the Saarland University Graduate School for Computer Science. JB received financial support from the Cluster of Excellence for Multimodal Computing and Interaction (MMCI) and is also grateful for support from the SDU2020 funding initiative at the University of Southern Denmark.

\section{Author details}

Graduate Program in Bioinformatics, Institute of Biological Sciences, Federal University of Minas Gerais (Universidade Federal de Minas Gerais), Belo Horizonte, Minas Gerais, Brazil. ${ }^{2}$ Institute of Biological Sciences, Federal University of Pará, Belém, Pará, Brazil. ${ }^{3}$ Department of Mathematics and Computer Science, University of Southern Denmark, Odense, Denmark. ${ }^{4}$ Computational Systems Biology, Max Planck Institute for Informatics, Campus E 2.1, 66123 Saarbrucken, Germany.

Received: 15 April 2014 Accepted: 14 May 2015

Published online: 11 June 2015

\section{References}

1. Babu MM, Lang B, Aravind L. Methods to reconstruct and compare transcriptional regulatory networks. Methods Mol Biol. 2009;541:163-80.

2. Rodionov DA. Comparative genomic reconstruction of transcriptional regulatory networks in bacteria. Chem Rev. 2007;107(8):3467-97.

3. Rottger R, Ruckert U, Taubert J, Baumbach J. How little do we actually know? On the size of gene regulatory networks. IEEE/ACM Trans Comput Biol Bioinform. 2012;9(5):1293-300.

4. Monot M, Honore N, Garnier T, Zidane N, Sherafi D, Paniz-Mondolfi A, et al. Comparative genomic and phylogeographic analysis of Mycobacterium leprae. Nat Genet. 2009:41(12):1282-9.

5. Dorella FA, Pacheco LG, Oliveira SC, Miyoshi A, Azevedo V. Corynebacterium pseudotuberculosis: microbiology, biochemical properties, pathogenesis and molecular studies of virulence. Vet Res. 2006;37(2):201-18.

6. Koffas M, Stephanopoulos G. Strain improvement by metabolic engineering: lysine production as a case study for systems biology. Curr Opin Biotechnol. 2005;16(3):361-6.

7. Moore DP, Klugman KP, Madhi SA. Role of Streptococcus pneumoniae in hospitalization for acute community-acquired pneumonia associated with culture-confirmed Mycobacterium tuberculosis in children: a pneumococcal conjugate vaccine probe study. Pediatr Infect Dis J. 2010;29(12):1099-04.

8. Baumbach J. CoryneRegNet 4.0 - A reference database for corynebacterial gene regulatory networks. BMC Bioinformatics. 2007:8:429.

9. Baumbach J, Brinkrolf K, Czaja LF, Rahmann S, Tauch A. CoryneRegNet: an ontology-based data warehouse of corynebacterial transcription factors and regulatory networks. BMC Genomics. 2006;7:24.

10. Baumbach J, Wittkop T, Kleindt CK, Tauch A. Integrated analysis and reconstruction of microbial transcriptional gene regulatory networks using CoryneRegNet. Nat Protoc. 2009;4(6):992-1005.

11. Baumbach J, Wittkop T, Rademacher K, Rahmann S, Brinkrolf K, Tauch A. CoryneRegNet 3.0-an interactive systems biology platform for the analysis of gene regulatory networks in corynebacteria and Escherichia coli. J Biotechnol. 2007;129(2):279-89. 
12. Pauling J, Rottger R, Tauch A, Azevedo V, Baumbach J. CoryneRegNet 6.0-Updated database content, new analysis methods and novel features focusing on community demands. Nucleic Acids Res. 2012;40(Database issue):D610-4.

13. Krawczyk J, Kohl TA, Goesmann A, Kalinowski J, Baumbach J. From Corynebacterium glutamicum to Mycobacterium tuberculosis-towards transfers of gene regulatory networks and integrated data analyses with MycoRegNet. Nucleic Acids Res. 2009;37(14), e97.

14. Wittkop T, Emig D, Lange S, Rahmann S, Albrecht M, Morris JH, et al Partitioning biological data with transitivity clustering. Nat Methods. 2010;7(6):419-20.

15. Rottger R, Kalaghatgi P, Sun P, Soares Sde C, Azevedo V, Wittkop T, et al. Density parameter estimation for finding clusters of homologous proteins-tracing actinobacterial pathogenicity lifestyles. Bioinformatics. 2013;29(2):215-22.

16. Beckstette M, Homann R, Giegerich R, Kurtz S. Fast index based algorithms and software for matching position specific scoring matrices. BMC Bioinformatics. 2006;7:389.

17. Dehal PS, Joachimiak MP, Price MN, Bates JT, Baumohl JK, Chivian D, et al. MicrobesOnline: an integrated portal for comparative and functional genomics. Nucleic Acids Res. 2010;38(Database issue):D396-400.

18. Sayers EW, Barrett T, Benson DA, Bolton E, Bryant SH, Canese K, et al. Database resources of the National Center for Biotechnology Information. Nucleic Acids Res. 2010;38(Database issue):D5-16.

19. Galagan JE, Sisk P, Stolte C, Weiner B, Koehrsen M, Wymore F, et al. TB database 2010: overview and update. Tuberculosis (Edinb). 2010;90(4):225-35.

20. Galagan JE, Minch K, Peterson M, Lyubetskaya A, Azizi E, Sweet L, et al. The Mycobacterium tuberculosis regulatory network and hypoxia. Nature. 2013;499(7457):178-83.

21. Machanick P, Bailey TL. MEME-ChIP: motif analysis of large DNA datasets. Bioinformatics. 2011;27(12):1696-7.

22. Fontan $\mathrm{P}$, Aris $\mathrm{V}$, Ghanny $\mathrm{S}$, Soteropoulos $\mathrm{P}$, Smith I. Global transcriptional profile of Mycobacterium tuberculosis during THP-1 human macrophage infection. Infect Immun. 2008;76(2):717-25.

23. Baumbach J. On the power and limits of evolutionary conservation-unraveling bacterial gene regulatory networks. Nucleic Acids Res. 2010;38(22):7877-84.

24. Singh P, Cole ST. Mycobacterium leprae: genes, pseudogenes and genetic diversity. Future Microbiol. 2011;6(1):57-71.

25. Jungwirth B, Sala C, Kohl TA, Uplekar S, Baumbach J, Cole ST, et al. High-resolution detection of DNA binding sites of the global transcriptional regulator GlxR in Corynebacterium glutamicum. Microbiology. 2013;159(Pt 1):12-22.

26. Kim HJ, Kim TH, Kim Y, Lee HS. Identification and characterization of glxR, a gene involved in regulation of glyoxylate bypass in Corynebacterium glutamicum. J Bacteriol. 2004;186(11):3453-60.

27. Letek M, Valbuena N, Ramos A, Ordonez E, Gil JA, Mateos LM. Characterization and use of catabolite-repressed promoters from gluconate genes in Corynebacterium glutamicum. J Bacteriol. 2006;188(2):409-23.

28. Kohl TA, Baumbach J, Jungwirth B, Puhler A, Tauch A. The GlxR regulon of the amino acid producer Corynebacterium glutamicum: in silico and in vitro detection of DNA binding sites of a global transcription regulator. J Biotechnol. 2008;135(4):340-50.

29. Toyoda K, Teramoto H, Inui M, Yukawa H. Genome-wide identification of in vivo binding sites of GlXR, a cyclic AMP receptor protein-type regulator in Corynebacterium glutamicum. J Bacteriol. 2011;193(16):4123-33.

30. Gama-Castro S, Salgado H, Peralta-Gil M, Santos-Zavaleta A, Muniz-Rascado $\mathrm{L}$, Solano-Lira $\mathrm{H}$, et al. RegulonDB version 7.0: transcriptional regulation of Escherichia coli K-12 integrated within genetic sensory response units (Gensor Units). Nucleic Acids Res. 2011;39(Database issue):D98-105.

31. Salgado H, Gama-Castro S, Peralta-Gil M, Diaz-Peredo E, Sanchez-Solano F, Santos-Zavaleta A, et al. RegulonDB (version 5.0): Escherichia coli K-12 transcriptional regulatory network, operon organization, and growth conditions. Nucleic Acids Res. 2006;34(Database issue):D394-7.

32. Salgado H, Peralta-Gil M, Gama-Castro S, Santos-Zavaleta A, Muniz-Rascado L, Garcia-Sotelo JS, et al. RegulonDB v8.0: omics data sets, evolutionary conservation, regulatory phrases, cross-validated gold standards and more. Nucleic Acids Res. 2013;41(Database issue):D203-13.

33. Black GF, Thiel BA, Ota MO, Parida SK, Adegbola R, Boom WH, et al. Immunogenicity of novel DosR regulon-encoded candidate antigens of Mycobacterium tuberculosis in three high-burden populations in Africa. Clin Vaccine Immunol. 2009;16(8):1203-12.

\section{Submit your next manuscript to BioMed Central and take full advantage of:}

- Convenient online submission

- Thorough peer review

- No space constraints or color figure charges

- Immediate publication on acceptance

- Inclusion in PubMed, CAS, Scopus and Google Scholar

- Research which is freely available for redistribution

Submit your manuscript at www.biomedcentral.com/submit 